\section{P061 THE PREVALENCE AND MONITORING OF CIRRHOTIC AND NON-CIRRHOTIC CYSTIC FIBROSIS LIVER DISEASE IN AN ADULT WEST OF SCOTLAND COHORT}

Damien Leith*, Poppy Holland, Gordon MacGregor, Matthew Priest. Queen Elizabeth University Hospital, Glasgow, UK

\subsection{6/gutjnl-2021-BASL.70}

Cystic fibrosis $[\mathrm{CF}]$ is a systemic illness resulting from loss of function of the CFTR protein on secretory epithelium. CF liver disease [CFLD] remains under recognised, despite being the third leading cause of mortality in CF. ${ }^{1}$ This study determined the prevalence of CFLD and CFLD-cirrhosis in a cohort of adult CF patients. Adherence to local surveillance protocols for diagnosing and monitoring these conditions, including engagement of specialist hepatology services, was assessed.

The study identified 270 patients with a diagnosis of CF in the West of Scotland Adult Cystic Fibrosis Service. Electronic clinical records were reviewed to determine the prevalence of pre-existing diagnoses of CFLD and assess for evidence of cirrhosis in these patients. The study assessed if standards, as determined by local protocols, of liver monitoring and hepatology service involvement in non-CFLD, CFLD and CFLDcirrhosis patients were met.

The average age of the cohort was 32 years (range 16-71) and 145 were male. There was a pre-existing diagnosis of CFLD in $80 / 270$ patients (30\%), with evidence of cirrhosis in 23 patients (29\% of CFLD patients; 9\% of the total cohort). Of those with CFLD/CFLD-cirrhosis, regular follow up with or previous discharge from hepatology services occurred in $34 / 80$ patients $(43 \%)$.

The local standard of yearly liver function tests [LFT] in non-CFLD and non-cirrhotic CFLD patients and 6-monthly LFT in CFLD-cirrhosis patients was met in 241/270 individuals (89\%). The local standard of ultrasound 5 -yearly in nonCFLD patients, 2 yearly in non-cirrhotic CFLD patients and 6 monthly in CFLD-cirrhosis patients was met in 190/270 individuals (70\%). Of those with CFLD, 41/80 (51\%) had been assessed with Fibroscan. Of those with CFLD-cirrhosis 15/23 $(65 \%)$ had an AFP measured at any point with 2/23 (9\%) having had an AFP within 6 months and 17/23 (73\%) had received an OGD at any point. [See table 1].

CFLD is a source of significant morbidity and mortality in CF patients and affects $30 \%$ of our patient population, with $9 \%$ of our CF cohort having evidence of cirrhosis. Despite this, a risk of delayed or missed diagnosis has been identified due to non-CFLD patients not receiving LFT and USS monitoring as per local protocol. There is also significant room for improvement in ensuring those with CFLD and CFLD-cirrhosis are referred to hepatology services which would also assist in ensuring appropriate ongoing disease monitoring - particularly Fibroscan to aid diagnosis of CFLD-cirrhosis and ensure appropriate HCC and variceal surveillance.

\section{REFERENCE}

1. Debray D, Kelly D, Houwen R, Strandvik B, Colombo C. Best practice guidance for the diagnosis and management of cystic fibrosis-associated liver disease. $J$ Cyst Fibros 2011;10:29-36.

\section{P062 ASSESSMENT OF BONE MINERAL DENSITY IN PATIENTS REFERRED FOR ELECTIVE LIVER TRANSPLANTATION}

Hannah McDowell*, Michael Williams. Scottish Liver Transplant Unit, Royal Infirmary of Edinburgh, Edinburgh, UK

\subsection{6/gutjnl-2021-BASL.71}

Cirrhosis is known to be associated with an increased risk of osteoporosis and fractures, and current UK guidelines recommend bone mineral density assessment in all patients with cirrhosis. Liver transplantation is associated with a further temporary reduction in bone mineral density. We reviewed the notes for 219 patients who underwent liver transplantation at the Scottish Liver Transplant Unit between January 2014 and July 2020. The majority of patients $(85 \%)$ had never had a DEXA scan pre-transplant. Of the $15 \%$ who had had DEXA scans pre-transplant, only $21 \%$ had normal bone mineral density, with $42 \%$ having osteopenia and 36\% having osteoporosis. There was no significant difference in the pre-DEXA FRAX scores between patients who did and did not have DEXA scans, suggesting that we are not targeting investigations at those with the highest baseline risk. A further 60 patients underwent DEXA scan post-transplant: only $25 \%$ were normal, with 48\% having osteopenia and 27\% having osteoporosis. Patients with cholestatic liver disease were more likely to have had a DEXA scan than other aetiologies $(40 \%$ vs $9.5 \%, \mathrm{p}<0.001)$, although there was not a significant difference in the rates of osteoporosis (44\% vs $29 \%$, p0.74). This data confirms that patients with cirrhosis have a significant risk of osteoporosis but shows that this is being underinvestigated in patients subsequently requiring liver transplantation.

Abstract P061 Table 1 CFLD investigation, monitoring and hepatology referral by patient group

\begin{tabular}{|c|c|c|c|}
\hline & $\begin{array}{l}\text { Non-CFLD } \\
{[190 / 270 ; 70 \%]}\end{array}$ & $\begin{array}{l}\text { Non-Cirrhotic CFLD } \\
{[57 / 270 ; 21 \%]}\end{array}$ & $\begin{array}{l}\text { CFLD Cirrhosis } \\
\text { [23/270; } 9 \%]\end{array}$ \\
\hline LFT Standard Met & $173 / 190(91 \%)$ & $49 / 57(86 \%)$ & $19 / 23(83 \%)$ \\
\hline \multicolumn{4}{|c|}{ [Yearly LFT Non-CFLD; 6 monthly LFT CFLD/CFLD-cirrhosis] } \\
\hline Ultrasound Standard Met & $138 / 190(73 \%)$ & $35 / 57(61 \%)$ & $17 / 23(74 \%)$ \\
\hline \multicolumn{4}{|c|}{ [5-yearly US Non-CFLD; 2-yearly US Non-cirrhotic CFLD; 6-monthly US CFLD-cirrhosis } \\
\hline Gastroenterology Referral & N/A & $22 / 57(39 \%)$ & $12 / 23(52 \%)$ \\
\hline Fibroscan [At any time] & $17 / 190(9 \%)$ & $31 / 57(54 \%)$ & $10 / 23(43 \%)$ \\
\hline Endoscopy [At any time] & $27 / 190(14 \%)$ & $5 / 57(9 \%)$ & $17 / 23(73 \%)$ \\
\hline
\end{tabular}

